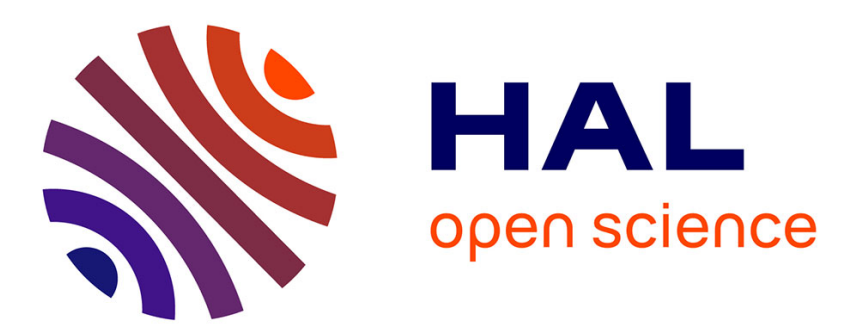

\title{
A Combined Time and Frequency Algorithm for Improved Channel Estimation in TDS-OFDM
}

Ming Liu, Matthieu Crussière, Jean-François Hélard

\section{To cite this version:}

Ming Liu, Matthieu Crussière, Jean-François Hélard. A Combined Time and Frequency Algorithm for Improved Channel Estimation in TDS-OFDM. IEEE International Conference on Communications (ICC 2010), May 2010, Cape Town, South Africa. pp.1-6, 10.1109/ICC.2010.5501781 . hal-00491817

\section{HAL Id: hal-00491817 https://hal.science/hal-00491817}

Submitted on 14 Jun 2010

HAL is a multi-disciplinary open access archive for the deposit and dissemination of scientific research documents, whether they are published or not. The documents may come from teaching and research institutions in France or abroad, or from public or private research centers.
L'archive ouverte pluridisciplinaire HAL, est destinée au dépôt et à la diffusion de documents scientifiques de niveau recherche, publiés ou non, émanant des établissements d'enseignement et de recherche français ou étrangers, des laboratoires publics ou privés. 


\title{
A Combined Time and Frequency Algorithm for Improved Channel Estimation in TDS-OFDM
}

\author{
Ming Liu, Matthieu Crussière, Jean-François Hélard \\ European University of Brittany (UEB), Institute of Electronics and Telecommunications of Rennes (IETR), \\ INSA-Rennes, France \\ Email: \{first name. last name\}@insa-rennes.fr
}

\begin{abstract}
In contrast to the classical cyclic prefix (CP)-OFDM, the time domain synchronous (TDS)-OFDM employs a known pseudo noise (PN) sequence as guard interval (GI). Conventional channel estimation methods for TDS-OFDM are only based on the PN sequence and consequently suffer from intersymbol interference. This paper proposes a novel two-stage channel estimation method which combines the estimation results from the PN sequence and, most importantly, the estimation results obtained from the OFDM data symbols. A simple feedback loop that excludes the channel decoder is employed for the OFDM data based estimation. The MMSE criteria is used to obtain the best combination results and an iterative process is proposed to progressively refine the estimation. Both MSE and BER simulations are carried out to prove the effectiveness of the proposed algorithm in the DTMB system which is based on TDS-OFDM signalling.
\end{abstract}

\section{INTRODUCTION}

Orthogonal frequency division multiplexing (OFDM) is widely used in several modern wireless communication systems such as wireless local area network (WLAN), digital audio/video broadcasting (DAB/DVB), etc. Usually, a cyclic prefix $(\mathrm{CP})$ is inserted as guard interval (GI) between two adjacent OFDM symbols. The CP not only prevents the intersymbol interference (ISI) but also converts the linear convolution between the transmitted signal and the channel impulse response (CIR) into a circular one. With the help of the $\mathrm{CP}$, the time domain channel convolution effect is indeed transformed into a set of parallel attenuations in the frequency domain, which simplifies the equalization process at the receiver. It was recently proposed to replace the $\mathrm{CP}$ by a known pseudo noise (PN) sequence. In contrast to the classical CP-OFDM, this OFDM technique using PN as GI is referred to as time domain synchronous (TDS)-OFDM [1]. The known PN sequence also serves as training sequence for channel estimation and synchronization. Unlike conventional CP-OFDM based systems, no pilots are inserted in the data symbols, thereby improving the spectrum efficiency. With such advantage, TDS-OFDM has been adopted in the Chinese digital television/terrestrial multimedia broadcasting (DTMB) system [2].

In TDS-OFDM, accurate channel estimation is quite important not only to enable coherent detection but also to prevent ISI between the OFDM data symbols. Since the estimated channel impulse response (CIR) is used to subtract the GI from the received TDS-OFDM signal, any inaccuracy in the CIR estimation will cause imperfect PN removal and consequentially introduce interference between the data symbols. Many approaches can be found in the literatures that attempt to solve this issue. [3] proposed to make channel estimation in time domain based on the correlation property of the PN sequence. Taking the advantage of the cyclic structure of the GI in the DTMB system, estimations from different observation windows are combined to get the final estimation result. In contrast, [4] proposed a frequency domain estimation algorithm. The channel estimation is based on the PN sequence, including its tail produced by the multipath channel. The channel estimation is refined by iteratively subtracting the current OFDM data symbols. However, since the PN is always contaminated by the ISI coming from the previous OFDM data symbols, the estimation based on the PN sequence suffers a substantial estimation error floor. To overcome this problem, [5] proposed to alternately remove the mutual interference between the PN sequence and the OFDM data symbols. However, the performance severely degrades in high frequency-selective channel. On the other hand, [6] proposed a decision directed channel estimation method. The data symbols which fall into a so-called decision region are hard decided and the resulting data symbols are treated as "training symbols". The final channel estimation is made only depending on these virtual training symbols. However, it has the potential risk that insufficient reliable training symbols can be obtained in low SNR scenarios or in the deep fading subbands. With such an approach, the channel can thus hardly be well estimated. An alternative solution, proposed by [7], is to carry out an iterative turbo channel estimation. The soft decoded data information output from the channel decoder is used to rebuild the transmitted data symbols which become accurate enough to serve as training symbols. However, the complexity as well as the time delay caused by the decoding and the interleaving process can reach prohibitive values for transmission schemes employing sophisticated forward error correction (FEC) blocks. For instance, the turbo algorithm of [7] can not be used in practice for the DTMB system in which an interleaver of depth 170 (or 510) OFDM symbols is used.

To solve these problems, we propose an effective two-stage channel estimation algorithm with low complexity. In the first stage, channel estimation is made through a classical time domain cross-correlation between the received PN sequence and local generated one. Only part of the GI (discarding the 


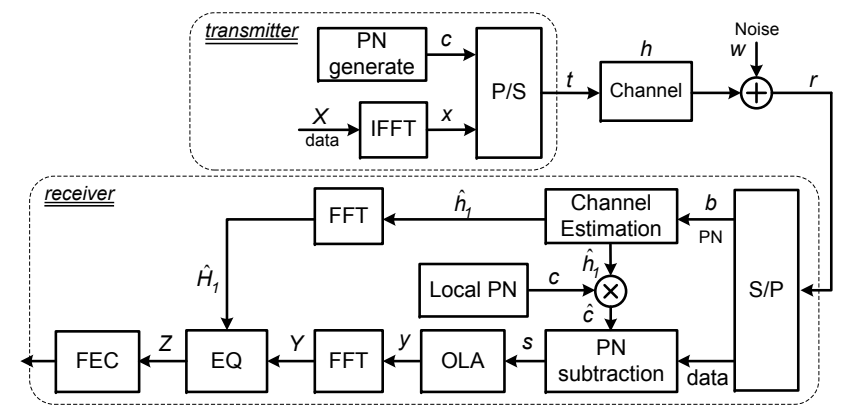

Fig. 1. Baseband model of the TDS-OFDM based system.

$\mathrm{CP}$ ) is used to reduce the ISI on the estimation. While in the second stage, channel estimation is obtained using the rebuilt data symbols in the frequency domain. In contrast to the full turbo-like channel estimation as shown in [7], we propose a data-aided channel estimation method with simple feedback loop, i.e. excluding the FEC decoding process. The data symbols are rebuilt based on the log-likelihood ratio (LLR) output from the soft demapper. The instantaneous channel frequency response (CFR) over all subcarriers can then be obtained by using the rebuilt data symbols as known training symbols. Averaging and interpolation processes are proposed to enhance the reliability of the estimation obtained from these virtual training symbols. In the final step, estimations from the two stages are combined according to the minimum mean squared error (MMSE) criteria.

The paper is organized as follows. In Section II, the TDS-OFDM signal model is described. The proposed twostage data-aided channel estimation algorithm is presented in Section III. Simulation results are shown in Section IV. Conclusions are drawn in Section V.

\section{TDS-OFDM SIGNAL MODEL}

The baseband model of the TDS-OFDM based system considered in this paper is presented in Fig. 1. The modulated complex data symbols are segmented into sequences of length $N$. The $i$ th data sequence is written as $\left\{X^{(i)}(k)\right\}_{k=0}^{N-1}$. Then, it is converted to the time domain by an $N$-point inverse fast Fourier transform (IFFT). The time domain data sequence $x^{(i)}(n)$ is written as:

$$
x^{(i)}(n)=\frac{1}{\sqrt{N}} \sum_{k=0}^{N-1} X^{(i)}(k) e^{j \frac{2 \pi}{N} n k}, 0 \leq n \leq N-1 .
$$

A PN sequence of length $\nu$ serving as GI, namely $\left\{c^{(i)}(n)\right\}_{n=0}^{\nu-1}$, is inserted before $x^{(i)}(n)$. The $i$ th transmitted OFDM symbol $t^{(i)}(n)$ is then:

$$
t^{(i)}(n)=\left\{\begin{array}{lc}
c^{(i)}(n) & -\nu \leq n<0 \\
x^{(i)}(n) & 0 \leq k \leq N-1
\end{array} .\right.
$$

After passing the multipath channel, the received signal $y^{(i)}(n)$ is expressed as:

$$
y^{(i)}(n)=t^{(i)}(n) * h^{(i)}(n)+w(n),
$$

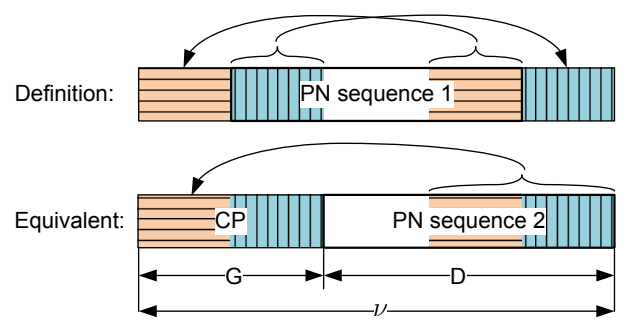

Fig. 2. Structure of the GI.

where $*$ denotes the linear convolution, $h^{(i)}(n)$ and $w(n)$ are the discrete CIR and additive white Gaussian noise (AWGN), respectively. The channel is assumed to be wide sense stationary uncorrelated scattering (WSSUS) and quasistatic, i.e. different paths are statistically uncorrelated and the CIR remains constant during at least one OFDM symbol. After removing the GI, the overlap-add (OLA) method is used to ensure the circular convolution between the data sequence and the CIR [8]. The received data sequence $r^{(i)}(n)$ then becomes:

$$
r^{(i)}(n)=x^{(i)}(n) \circledast h^{(i)}(n)+w^{\prime}(n), 0 \leq n \leq N-1,
$$

where $\circledast$ denotes the circular convolution, $w^{\prime}(n)$ is the noise after OLA and is thus slightly colored. According to the convolution theorem, the Fourier transform converts the time domain circular convolution into the frequency domain pointwise product:

$$
R^{(i)}(k)=H^{(i)}(k) \cdot X^{(i)}(k)+W(k), 0 \leq k \leq N-1,
$$

where $R, H$ and $W$ are $N$-point Fast Fourier transform (FFT) of $r, h$ and $w^{\prime}$, respectively. $H$ is the CFR. Equation (5) shows that the received TDS-OFDM symbols can be equalized by a simple one-tap equalizer applied in the frequency domain. For the notation simplicity, OFDM symbol index $i$ is omitted in the following sections.

\section{Proposed Combined Time And Frequency Channel Estimation Method}

A two-stage channel estimation algorithm is proposed in this section. A time domain circular cross-correlation between the received PN and a local generated one is used to estimate the CIR. A low-complexity data-aided channel estimation is then proposed for the second estimation stage. The final channel estimation is the MMSE combination of the two estimations.

\section{A. Initial Time Domain Channel Estimation}

The initial channel estimation is obtained from the exploitation of the PN in the GI. In the DTMB system, the $\nu$-length GI consists of a $D$-length maximum length sequence (msequence) as well as its pre- and post- circular extensions as shown in Fig. 2. Since any time shift of an m-sequence is itself an m-sequence [9], the GI can also be treated as another $D$-length m-sequence, denoted as $p(n)$, with its $G$-length CP. When the CIR is shorter than this CP, the circular convolution between the CIR and the PN sequence is held. After removing 
the $\mathrm{CP}$ of the $\mathrm{PN}$, the received $\mathrm{PN}$ sequence $b(n)$ is written as:

$$
\begin{aligned}
b(n) & =p(n) \circledast h(n)+w(n) \\
& =\sum_{l=0}^{L-1} h(l) p(n-l)_{D}+w(n), \quad 0 \leq n<D
\end{aligned}
$$

where $(\cdot)_{D}$ denotes modular $D$ operation. The circular crosscorrelation (denoted as $\otimes$ ) between the received PN sequence and the local generated one is performed as:

$$
\begin{aligned}
& R(n)=p(n+m) \otimes b(m) \\
= & p(n+m) \otimes[p(m) \circledast h(m)]+p(n+m) \otimes w(m) \\
= & {[p(n+m) \otimes p(m)] \circledast h(m)+p(n+m) \otimes w(m)(7) }
\end{aligned}
$$

Using the autocorrelation property of the PN sequence [9], $C_{p}(n)=\frac{1}{D} p(n+m) \otimes p(m)=\left\{\begin{array}{cc}1 & n=0 \\ -\frac{1}{D} & 0<n<D\end{array}\right.$,

a straightforward time domain channel estimator is chosen as:

$$
\begin{aligned}
\hat{h}(n)= & \frac{1}{D} R(n)=C_{p}(n) \circledast h(m)+\frac{1}{D} p(n+m) \otimes w(m) \\
= & h(n)-\underbrace{\frac{1}{D} \sum_{\substack{m=0 \\
m \neq n}}^{L-1} h(m)}_{\text {self interference }}+\underbrace{\frac{1}{D} \sum_{q=0}^{D-1} p(q) w(q+n),}_{\text {noise }} \\
& 0 \leq n<D .
\end{aligned}
$$

When the PN is sufficiently long, i.e. $-\frac{1}{D} \approx 0$, the normalized autocorrelation function $C_{p}$ is approximately Kronecker delta function. The estimator is asymptotically unbiased as $D \rightarrow \infty$. Since the CIR length $L$ can be estimated, the CIR estimation is truncated to a length of $L$, which will reduce the noise contribution with a ratio of $\frac{L}{D}$. Using the WSSUS assumption, the MSE of the CIR estimation is:

$$
\begin{aligned}
\text { mse }_{\hat{h}} & =\frac{1}{L} \sum_{l=0}^{L-1} E\left\{|h(l)-\hat{h}(l)|^{2}\right\} \\
& =\frac{L-1}{D^{2} L}+\frac{1}{D} \sigma_{w}^{2} .
\end{aligned}
$$

The first term is caused by the self interference of the PN sequence and is reciprocally proportional to the square of the PN length. The self interference can be neglected at low SNR and becomes an estimation error floor at high SNR. To improve the estimator (9), the self interference can partially be removed using the estimated CIR as:

$$
\begin{gathered}
\hat{h}^{\prime}(n)=\hat{h}(n)+\frac{1}{D} \sum_{\substack{m=0 \\
m \neq n}}^{L-1} \hat{h}(m) \\
=h(n)-\frac{1}{D^{2}} \sum_{\substack{m=0 \\
m \neq n}}^{L-1} \sum_{\substack{l=0 \\
l \neq m}}^{L-1} h(l)+\frac{1}{D} \sum_{q=0}^{D-1} p(q) n(q+n) \\
+\frac{1}{D^{2}} \sum_{\substack{m=0 \\
m \neq n}}^{L-1} \sum_{\substack{D-1 \\
m=0}}^{D-1} p(u) n(u+m), \quad 0 \leq n<D, \quad(11)
\end{gathered}
$$

yielding a new MSE:

$$
\begin{gathered}
m s e_{\hat{h}^{\prime}}=\frac{1}{L} \sum_{l=0}^{L-1} E\left\{\left|h(l)-\hat{h}^{\prime}(l)\right|^{2}\right\} \\
=\frac{1}{D} \sigma_{w}^{2}+\frac{(L-1)}{D^{3}} \sigma_{w}^{2}+\frac{(L-2)(L-1)^{2}}{D^{4} L} .
\end{gathered}
$$

Comparing (12) with (10), the estimation error floor is reduced by a ratio of about $\left(\frac{L}{D}\right)^{2}$. The initial CIR estimation (9) and (11) can eventually be expressed in a general form as:

$$
\hat{h}_{1}(n)=h(n)+\varepsilon_{1}(n),
$$

where $\varepsilon_{1}$ is the CIR estimation error. The estimation MSE of $\hat{h}_{1}(n)$ (denoted as $m s e_{\hat{h}_{1}}$ ) is given by (10) or (12). The CFR estimation $\hat{H}_{1}$ is finally obtained through an $N$-point FFT applied on $\hat{h}_{1}(n)$ and the corresponding MSE is:

$$
\begin{aligned}
& M S E_{\hat{H}_{1}}=\frac{1}{N} \sum_{k=0}^{N-1} E\left\{\left|H(k)-\hat{H}_{1}\right|^{2}\right\} \\
= & \frac{1}{N} \sum_{k=0}^{N-1} \sum_{n_{1}=0}^{L-1} \sum_{n_{2}=0}^{L-1} E\left\{\varepsilon_{1}\left(n_{1}\right)^{*} \varepsilon_{1}\left(n_{2}\right)\right\} e^{-j 2 \pi \frac{k}{N}\left(n_{2}-n_{1}\right)} .
\end{aligned}
$$

From (10) and (12), $E\left\{\varepsilon_{1}\left(n_{1}\right)^{*} \varepsilon_{1}\left(n_{2}\right)\right\}=0$ when $n_{1} \neq n_{2}$ in the error-floor-free region. The MSE of $\hat{H}_{1}$ then becomes:

$$
\begin{aligned}
M S E_{\hat{H}_{1}} & =\frac{1}{N} \sum_{k=0}^{N-1} \sum_{n_{1}=0}^{L-1} E\left\{\left|\varepsilon_{1}(n)\right|^{2}\right\} \\
& =L \cdot m s e_{\hat{h}_{1}} .
\end{aligned}
$$

\section{B. Improved Data-aided Channel Estimation}

1) Interference Analysis: To evaluate the MSE of the second stage estimation, we first need to analyze the interference introduced by the inaccuracy of the CIR estimation. Since the initial CIR estimation $\hat{h}_{1}(n)$ is used to subtract the GI from the TDS-OFDM signal, the CIR estimation error causes imperfect PN sequence removal. Then, the OLA is performed to restore the orthogonality between subcarriers. The residual PN sequence thus introduces interference in the data symbols which can be written as:

$$
y_{I}(n)=\sum_{l=0}^{L-1} \varepsilon_{1}(l) p(n-l), \quad 0 \leq n \leq \nu+L-1 .
$$

In the frequency domain, this interference is given by:

$$
\begin{gathered}
Y_{I}(k)=\frac{1}{\sqrt{N}} \sum_{n=0}^{N-1} y_{I}(n) e^{-j \frac{2 \pi}{N} n k} \\
=\frac{1}{\sqrt{N}} \sum_{n=0}^{\nu+L-1} \sum_{l=0}^{L-1} \varepsilon_{1}(l) p(n-l) e^{-j \frac{2 \pi}{N} n k} .
\end{gathered}
$$

When the GI is long enough, the interference can be approximated as a Gaussian variable according to the central limit 


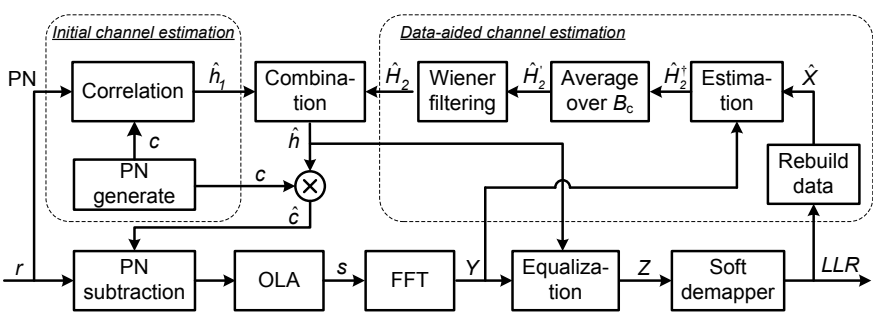

Fig. 3. Block diagram of the proposed data-aided channel estimation method.

theorem. Using (16) and employing the WSSUS assumption, power spectrum density of the interference is:

$$
\begin{aligned}
& N_{I}(k)=E\left\{Y_{I}^{*}(k) Y_{I}(k)\right\} \\
= & \frac{1}{N} \sum_{l=0}^{L-1} m s e_{\hat{h}_{1}}(l) \sum_{n=0}^{\nu+L-1} p^{*}(n-l) p(n-l) \\
= & \frac{\nu L}{N} m s e_{\hat{h}_{1}},
\end{aligned}
$$

where $(\cdot)^{*}$ denotes the complex conjugate.

2) Data-aided Channel Estimation: Fig. 3 presents the block diagram of the proposed data-aided channel estimation method. As demonstrated in previous sections, the received data can be written in the frequency domain as:

$$
Y(k)=H(k) \cdot X(k)+I(k)+W(k),
$$

where $I$ is the interference caused by the residual PN sequence. At first, the signal is equalized using the initial CFR estimation:

$$
Z(k)=\frac{Y(k)}{\hat{H}_{1}(k)} .
$$

The soft-output demapper then computes the LLR of the bits belonging to each complex data symbol. The estimated data symbol $\hat{X}(k)$ on the $k$ th subcarrier can be computed by:

$$
\hat{X}(k)=\sum_{\alpha_{i} \in \Psi} \alpha_{i} \cdot P\left(X(k)=\alpha_{i}\right),
$$

where $\alpha_{i}$ is a constellation point, $\Psi$ is the set of the constellation points of a given modulation scheme. $P\left(X(k)=\alpha_{i}\right)$ is the probability when the transmitted symbol is $\alpha_{i}$ and can be computed as the product of the probabilities of all the bits belonging to this constellation point:

$$
P\left(X(k)=\alpha_{i}\right)=\prod_{l=1}^{\log _{2}|\Psi|} P\left(b_{l} \mid X(k)=\alpha_{i}\right),
$$

where $|\Psi|$ is the cardinality of the set of constellation points, $b_{l} \in\{0,1\}$ is the $l$-th bit in a complex data symbol. The conditional probability $P\left(b_{l} \mid X(k)=\alpha_{i}\right)$ can be directly computed from the LLR of the $l$ th bit in the symbol $X(k)$.

Specifically, in the case of a Gray-coded QPSK, the data symbol is computed by:

$$
\hat{X}(k)=\frac{1}{\sqrt{2}}\left[\tanh \left(\frac{L L R_{1}(k)}{2}\right)+j \tanh \left(\frac{L L R_{2}(k)}{2}\right)\right],
$$

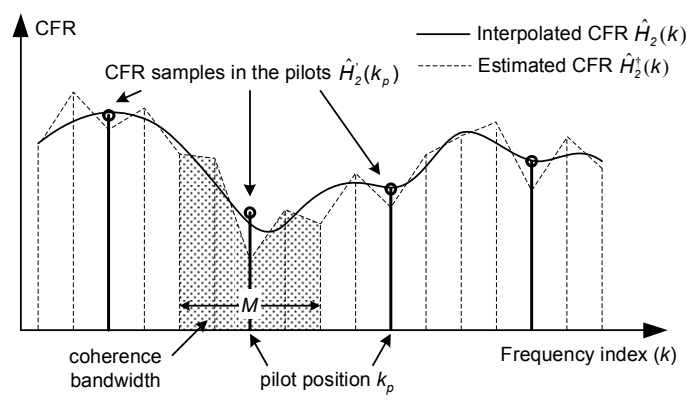

Fig. 4. Averaging and interpolation.

where $L L R_{1}(k)$ and $L L R_{2}(k)$ are respectively the first and second LLR output from the soft demapper corresponding to the input complex data symbol $Z(k)$. Then, the second channel estimation is simply derived as:

$$
\hat{H}_{2}^{\dagger}(k)=\frac{1}{E_{\alpha}} \hat{X}(k)^{*} \cdot Y(k),
$$

where $E_{\alpha}$ is the power of the constellation point. $\hat{H}_{2}^{\dagger}(k)$ is the instantaneous data-aided channel estimation.

3) Averaging and Wiener Filtering: The proposed averaging and interpolation approaches can be interpreted in Fig.4. In contrast to a full iterative turbo channel estimation as [7], the proposed method does not include any error correction before rebuilding the data. So the instantaneous estimation $\hat{H}_{2}^{\dagger}$ is corrupted by the noise and the channel fading. Considering that the channel frequency response does not significantly vary within the coherence bandwidth [10], $\hat{H}_{2}^{\dagger}$ is averaged over the coherence bandwidth to reduce the noise effect and improve the estimation. Defining a set $\Xi$ of virtual pilots indices and a set $\Theta_{k_{p}}$ of subcarrier indices within the coherence bandwidth with the $k_{p}$ th subcarrier as its central frequency, the CFR estimation samples in the virtual pilots, namely $\hat{H}_{2}^{\prime}\left(k_{p}\right)$, can be written as:

$$
\begin{aligned}
& \hat{H}_{2}^{\prime}\left(k_{p}\right)=\frac{1}{M} \sum_{m \in \Theta_{k_{p}}} \hat{H}_{2}^{\dagger}(m) \\
\approx & H\left(k_{p}\right)+\frac{1}{M} \sum_{m \in \Theta_{k_{p}}} \hat{X}(m)^{*}[I(m)+W(m)],
\end{aligned}
$$

where $k_{p} \in \Xi$. The cardinalities of $\Xi$ and $\Theta_{k_{p}}$ are $K$ and $M$, respectively. The $k_{p}$ 's should be selected according to the sampling theorem with $2 \times$ oversampling ratio [11]. From (24), the variance of the estimation error can be estimated as:

$$
\begin{aligned}
& \varepsilon_{2}(k)=E\left\{\left|H(k)-\hat{H}_{2}^{\prime}(k)\right|^{2}\right\} \\
= & \frac{\sigma_{W}^{2}+N_{I}}{M^{2}} \sum_{m \in \Theta_{k_{p}}}|\hat{X}(m)|^{2}=\frac{\sigma_{W}^{2}+N_{I}}{M} \bar{E}_{\hat{X}}\left(k_{p}\right)(25)
\end{aligned}
$$

where $\bar{E}_{\hat{X}}\left(k_{p}\right)$ is the mean power of the rebuilt data symbols within $\Theta_{k_{p}}$. Finally, to obtain the $N$-length CFR estimation $\hat{H}_{2}$, a frequency domain Wiener filtering [11] is performed on the CFR estimation samples i.e. $\hat{H}_{2}^{\prime}\left(k_{p}\right)$ 's available in $\Xi$ :

$$
\hat{H}_{2}(k)=\sum_{k_{p} \in \Xi} f\left(k, k_{p}\right) \hat{H}_{2}^{\prime}\left(k_{p}\right), \quad 0 \leq k<N,
$$


where $f\left(k, k_{p}\right)$ 's are the Wiener filter coefficients computed to minimize $E\left\{\left|H(k)-\hat{H}_{2}(k)\right|^{2}\right\}$. The MMSE solution of this problem is:

$$
f^{T}=\Phi^{-1} \theta
$$

where $\Phi=E\left\{\hat{H}_{2}^{\prime} \hat{H}_{2}^{\prime}\right\}$ is the $K \times K$ autocorrelation matrix of observation vector $\hat{H}_{2}^{\prime}, \theta=E\left\{\hat{H}_{2}^{\prime} H\right\}$ is the $K \times N$ crosscorrelation matrix of $\hat{H}_{2}^{\prime}$ and desired output $H$. Note that the coefficients can be computed in either non-adaptive [11] or adaptive manners [12]. The MSE of the estimation $\hat{H}_{2}(k)$ is:

$$
M S E_{\hat{H}_{2}}(k)=\lambda_{H}^{2}(k)-\theta_{k}^{T} \Phi^{-1} \theta_{k}^{*}
$$

where $\lambda_{H}^{2}$ is the variance of the desired response $H$.

\section{Combination}

When the two channel estimations from the two different sources are obtained, we finally propose to carry out a weighted combination:

$$
\hat{H}=\beta \hat{H}_{1}+(1-\beta) \hat{H}_{2} .
$$

The MMSE criteria is used to determine the optimal weighting factor $\beta_{\text {opt }}$. Considering (14) and (28), the MSE of the combined estimation is:

$$
\mathcal{J}=E\left\{|H-\hat{H}|^{2}\right\}=\beta^{2} M S E_{\hat{H}_{1}}+(1-\beta)^{2} M S E_{\hat{H}_{2}} .
$$

Since the MSE function $\mathcal{J}$ is convex, the $\beta_{\text {opt }}$ is found when setting the derivative of $\mathcal{J}$ with respect to $\beta$ equal to zero i.e. $\frac{\partial \mathcal{J}}{\partial \beta}=0$. It can be obtained that

$$
\beta_{\text {opt }}=\frac{M S E_{\hat{H}_{2}}}{M S E_{\hat{H}_{1}}+M S E_{\hat{H}_{2}}} .
$$

The channel estimation result is then further refined in an iterative way. The combined estimation result (29) is used as the initial channel estimation to subtract the PN and equalize data in the next iteration. With a better initial channel estimation, the data aided channel estimation can thus be improved. The iteration terminates when the channel estimation is sufficiently good or the maximum iteration times is achieved.

\section{Simulation Results}

The simulation parameters are chosen according to the DTMB system [2]. Specifically, the length of the GI is 420. The interleaver is set to the $(52,240)$ mode. The modulation scheme is 4QAM and the LDPC with code rate 0.8 is used. The COST 207 Typical Urban 6 paths (TU-6) channel model [13] is employed in the simulations. Moreover, simulations are also carried out in the single frequency network (SFN) scenario which is a spectrum efficient solution widely used in broadcast networks. In this case, same signals are sent from two different transmitters at the same time on the same carrier frequency. The signals from these two transmitters experience independent fadings. As shown in Fig. 5, the equivalent CIR of the SFN channel is the combination of the CIR's of two independent TU-6 channels. The propagation distance difference between the two signals transmitted from the two transmitters

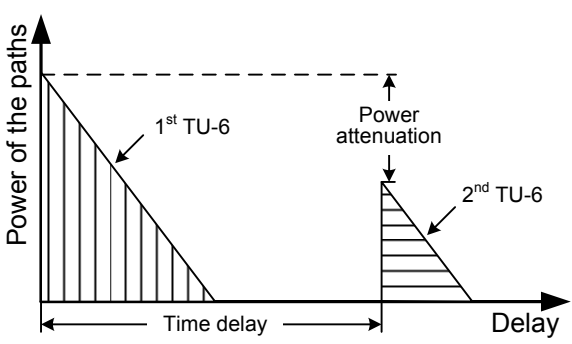

Fig. 5. CIR of the SFN channel.

will cause time delay and power attenuation on the second CIR and thus produces strong fading. In the following simulations, the distance difference is set to $8 \mathrm{~km}$ corresponding to $26.67 \mu \mathrm{s}$ time delay which makes the overall length of the CIR longer than that of the CP of the PN. The power attenuation is set to $10 \mathrm{~dB}$. The velocity is $30 \mathrm{~km} / \mathrm{h}$ in both SFN and non-SFN cases. Following the computation in [10], when the frequency correlation function is 0.9 , the coherence bandwidth of the TU-6 channel and the SFN channel are $18.8 \mathrm{kHz}$ and 2.58 $\mathrm{kHz}$, respectively. With the $2 \mathrm{kHz}$ subcarrier spacing in the DTMB system, the averaging length $M$ in (24) is accordingly set to 9 and 3 which is a good trade-off between the noise suppression and similarity of $\hat{H}_{2}^{\dagger}$ 's. The spacing between two adjacent pilots is also set to $M$ which will fully utilize all data subcarriers while fulfilling the sampling criteria in [11].

Fig. 6 and 7 present the MSE of the proposed algorithm in the TU-6 and the SFN channels, respectively. As mentioned in section III.C, the initial channel estimation results can be iteratively refined by the data-aided method. However, as evident from the results in Fig. 6, the estimation performance is already significantly improved after only the first dataaided iteration. That means the proposed method can provide satisfactory performance even without any iterative process. With the TU-6 channel, the iterative estimation converges after two iterations. The proposed method provides $6.8 \mathrm{~dB}$ gain over the PN based initial channel estimation method in terms of required SNR to achieve a MSE level of $10^{-3}$. The performance of the algorithm in [5] is also given as a reference which is well suited for low time-dispersive channels but suffers from ISI in the case of long echoes. It can then be seen that the proposed method outperforms method [5] in the TU-6 channel. On the other hand, in the SFN channel, since the CIR is longer than the CP of the PN, the interference from the previous OFDM symbol introduces a significant estimation error floor in the initial estimation. With the iterative data-aided channel estimation process, the error floor is progressively decreased. After three iterations, the estimation error floor can be reduced more than ten times. As method [5] is not adapted to the SFN channel which is strongly time-dispersive, its performance is not given.

The bit error rate (BER) of the DTMB system is also given to present the system level evaluation. The iteration times is set to 3 for the data-aided channel estimation. As shown in Fig. 8, in the TU-6 channel, the proposed data-aided channel 


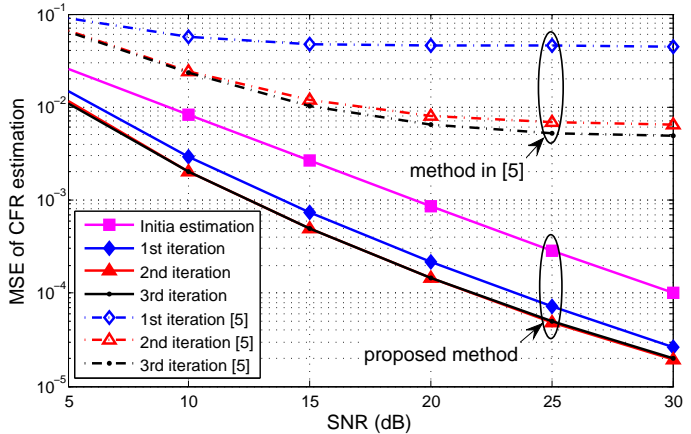

Fig. 6. MSE comparison in the TU-6 channel.

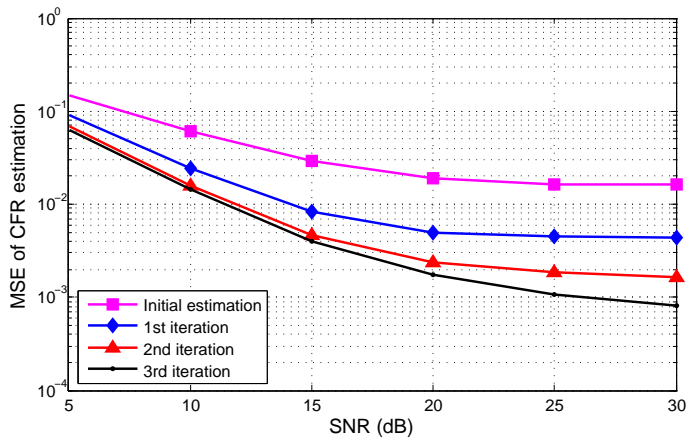

Fig. 7. MSE comparison in the SFN channel.

estimation method brings $1.7 \mathrm{~dB}$ and $0.4 \mathrm{~dB}$ gain over method [5] and the PN based initial estimation, respectively, in terms of required SNR to achieve BER of $5 \times 10^{-5}$. Moreover, the obtained BER performance is very close to the perfect channel estimation case. In the SFN channel, the improvement is even more significant as shown in Fig. 9. More than $1.7 \mathrm{~dB}$ gain over the PN based initial estimation method can be obtained using the proposed method. The loss due to imperfect channel estimation is limited to about $1 \mathrm{~dB}$ with respect to the ideal estimation case.

\section{CONClusion}

A novel two-stage channel estimation method for TDSOFDM is proposed in this paper. The initial channel estimation is made in the time domain using PN sequence in the GI, while the second estimation is obtained using the rebuilt data symbols in the frequency domain. Averaging within coherence bandwidth and Wiener filtering interpolation are used to improve the second estimation. The final channel estimation is obtained by combining the estimation results of the two stages according to MMSE criteria. Iterative process can be carried out to progressively refine the estimation results. The MSE and BER simulations show that the proposed dataaided channel estimation method significantly outperforms the classical PN based channel estimation method.

\section{REFERENCES}

[1] J. Wang, Z. Yang, C. Pan, M. Han, L. Yang, "A Combined Code Acquisition and Symbol Timing Recovery Method for TDS-OFDM," IEEE Trans. on Broadcasting, Vol. 49, pp. 304-308, 2003

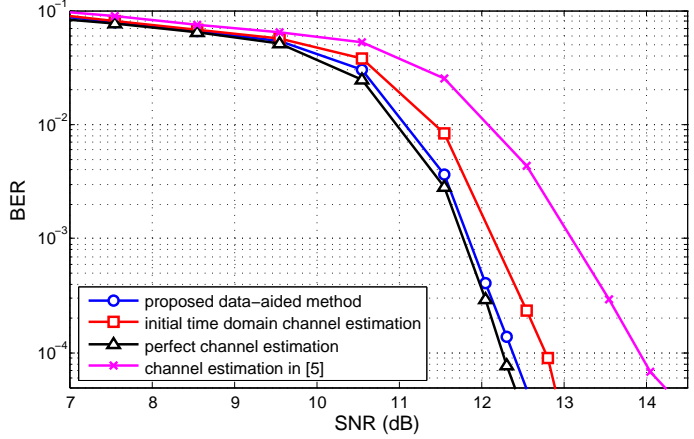

Fig. 8. BER comparison in the TU-6 channel.

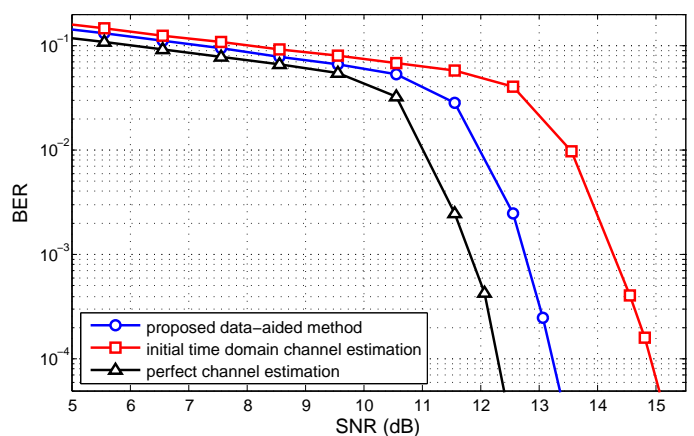

Fig. 9. BER comparison in the SFN channel.

[2] Framing Structure, Channel Coding and Modulation for Digital Television Terrestrial Broadcasting System, Chinese National Standard GB206002006.

[3] B. Song, L. Gui, Y. Guan, W. Zhang, "On channel estimation and equalization in TDS-OFDM based terrestrial HDTV broadcasting system," IEEE Trans, Consum. Electron., vol. 51, no. 3, pp. 790-797, Aug. 2005.

[4] S. Tang, F. Yang, K. Peng, C. Pan, K. Gong, Z. Yang, "Iterative Channel Estimation for Block Transmission with Known Symbol Padding - A New Look at TDS-OFDM,' in Proc. of the IEEE GLOBECOM'07, 2007, pp. 4269-4273.

[5] S. Tang, K. Peng, K. Gong, J. Song, C. Pan, Z. Yang, "Novel DecisionAided Channel Estimation for TDS-OFDM Systems," in Proc. of the IEEE ICC'08, 2008, pp. 946-950.

[6] F. Yang, K. Peng, J. Song, C. Pan, Z. Yang, "Novel Decision-Directed Channel Estimation Method for TDS-OFDM System", in Proc. of the IEEE International Conference on Communication Systems, 2008.

[7] M. Zhao, Z. Shi, M. C. Reed, "Iterative Turbo Channel Estimation for OFDM System over Rapid Dispersive Fading Channel," IEEE Trans. Wireless Commun., vol. 7, no.8, pp.3174-3184, Aug. 2008.

[8] M. Liu, M. Crussière, J.-F. Hélard, O. Pasquero, "Analysis and Performance Comparison of DVB-T and DTMB Systems for Terrestrial Digital TV," in Proc. of the IEEE International Conference on Communication Systems, 2008.

[9] D. Sarwate, M. Pursley, "Crosscorrelation properties of pseudorandom and related sequences," in Proc. of the IEEE, Vol.68, No.5, pp.593-619, 1980.

[10] T. Rappaport, Wireless Communications: Principles and Practice, 2nd Edition. Prentice-Hall, 2001.

[11] P. Hoeher, S. Kaiser, P. Robertson, "Two-Dimensional Pilot-SymbolAided Channel Estimation By Wiener Filtering," in Proc. of IEEE ICASSP'97, Munich, Germany, April 1997, pp. 1845-1848.

[12] C. Sgraja and J. Lindner, "Estimation of rapid time-variant channels for OFDM using Wiener filtering," in Proc. ICC'03, vol. 4, 2003, pp. 2390-2395.

[13] COST 207, "Digital land mobile radio communications," Final Report, 1989. 